\title{
VIRTUAL HIDDEN NETWORK
}

\author{
NATORI Shin \\ Department of Information Science, Faculty of Science, University of Tokyo \\ natori@is.s.u-tokyo.ac.jp
}

\begin{abstract}
In this paper, we first propose a new type of network model named Virtual Hidden Network (VHN), an invisible network layered over steganography channels. Unlike ordinary steganography systems, it can provide a secret communication channel between two persons who do not communicate directly. Next, we introduce a new steganography channel, TCP initial sequence number channel, the safety of which depends on the unpredictability of TCP initial sequence numbers. Finally, we show a prototype implementation of VHN over TCP initial sequence number channels and evaluate its safety and usability.
\end{abstract}

Keywords: Steganography, Information Hiding

\section{INTRODUCTION}

The recent proliferation of digital communication increases the importance of information security significantly. Cryptography, the art and science of protecting message, is now playing a central role in information security. Steganography, the art and science of hiding the existence of message itself, is also getting important, since it can provide a security layer that can not be provided by cryptography. For example, a cryptography system can encrypt a message so as not to leak the contents of hidden message to eavesdroppers, but the system can not hide the sender, recipient and the timing of communication. All eavesdroppers can notice the existence of some secret between the sender and recipient. On the other hand, a steganography system can hide a secret message into another innocent-looking message so as not to draw any suspicion of the existence of hidden message. This type of communication system can resist forenamed traffic analysis.

The security of a steganography system is the difficulty for the eavesdroppers to detect the existence of hidden message. If a steganography system is secure enough, no eavesdropper can detect the existence of 
hidden message. This means that if there exists a practically secure steganography system, it will decrease the effect of cryptography regulation significantly. Therefore, steganography is also important for the measurement of cost-effectiveness of cryptography regulation.

Most of the steganography systems so far proposed hide secret messages into innocent-looking cover message by replacing some noisy region of the cover message (such as low order bits of digitized photographs) with encrypted secret message. The security of these steganography systems depends on the indistinguishability between the noisy region of cover data and encrypted message. There is no theoretical guarantee of the randomness of the noisy region, and many such steganography systems were turned out to be vulnerable to careful eavesdroppers [7, 13]. Some steganography systems have provable safety, but they provide only small bandwidth and can not accommodate practical messages $[12,5]$. If one want to send his message with provable secrecy, he should fragment his message into small parts and use such systems many times. This may change the traffic pattern between sender and recipient, and may eventually cause suspicion of the existence of some secret [11].

In this paper, we introduce a new steganographic network model, Virtual Hidden Network (VHN), an invisible network layered over steganography channels. It amplifies the bandwidth of secret communication of steganography by utilizing not only the steganography channel between sender and recipient but also the otherwise discarded steganography channels between the nodes in it. When a sender want to send a secret message to a recipient in VHN, the sender first send his message to one of his neighbor nodes through an underlying steganography channel. The neighbor node selects one of its neighbor node as next node and forward the message to it. This forwarding is repeated until the message reaches the recipient. If the underlying steganography system is secure enough, no eavesdropper can notice the relationship between the sender and recipient, since no direct communication is needed between them.

In order to construct a practical VHN, one should carefully choose a steganography system as its underlying layer. At least, the steganography system should be able to hide and send messages automatically. In this paper, we introduce one such steganography channel, TCP initial sequence number channel, a steganography channel that resides in initial sequence number part of TCP connections. Although its bandwidth is small, it is secure and can deliver secret messages automatically. We amplify its bandwidth with VHN and show a way to construct a secure, invisible messaging network over the Internet.

This paper is organized as follows. Section 2 briefly describes related works. Section 3 introduces the definition of VHN and its protocols. Sec- 
tion 4 briefly describes a steganographic channel in TCP initial sequence numbers. Section 5 presents a prototype implementation of VHN using TCP initial sequence number channel. Section 6 contains conclusions.

\section{RELATED WORKS}

Onion Routing is a network framework that provides resistance to traffic analysis [9]. It uses many onion routers to provide anonymity. Each packet the sender want to send passes through a sequence of onion routers. Each onion router decrypts (or encrypts) the received packet and forward it to next onion router. It is difficult to trace the packets on an onion routing network, since all packets are alike (they are all encrypted and have the same length) and the sequence of onion routers a packet go through is kept secret. The sequence is kept secret to even onion routers themselves and each onion router knows only the decryption key and next onion router. Therefore, the security of Onion Routing network can not be corrupted even by intruding into some of onion routers.

Virtual Private Network (VPN) is virtual, secure network over public network [3]. It usually interconnects private networks with some tunneling protocol. VPN usually uses cryptography to protect the virtual network from eavesdropping, tampering, and impersonating.

VHN is similar to these notions, but its primary intention, hiding the existence of entire virtual network, is out of the scope of these notions. VHN can be regarded as a "concealed" version of VPN, or a distant variant of Onion Routing architecture that provides anonymity by hiding the secret message into another message, not crowding messages from many sources.

\section{VIRTUAL HIDDEN NETWORK 3.1. DEFINITION OF VHN}

A Virtual Hidden Network is a connected network whose edges are steganography channels (Figure 1). Its nodes are communication entities, such as hosts or network users. The steganography channels are usually bidirectional and transfer secret messages between two nodes. Each node may or may not forward received messages. When and how a message is forwarded depends on the protocol the VHN adopts.

The structure of VHN is analogous to that of the IP (Internet Protocol [4]) network (Figure 2). In both cases the upper layer transmits messages to destination using lower layer transmission channel several 


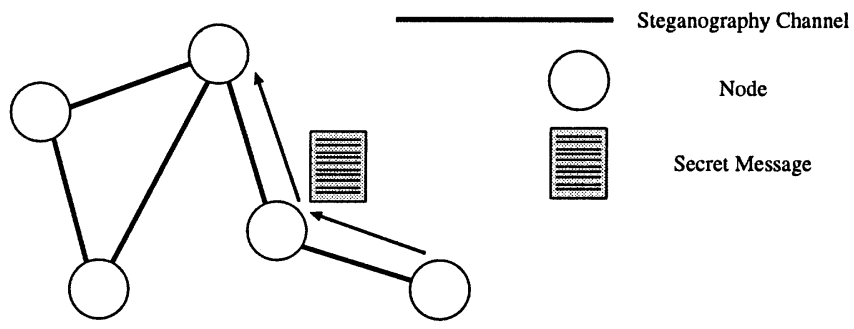

Figure 1 Virtual Hidden Network

times. Here data link network means local network, such as Ethernet or PPP connection.

\begin{tabular}{|c|c|c|}
\hline IP Network & Network Layer & VHN \\
\hline Data Link Network & Data Link Layer & Steganography Channel \\
\hline
\end{tabular}

Figure 2 Analogy between IP Network and VHN

The fundamental difference between IP Network and VHN is that the data link network the former uses can send arbitrary packet at any given time, but the steganography channel the latter uses can send a packet only when a cover message is actually sent. So the latter can forward message only asynchronously. This forwarding may take long time, and in general case there is not easy way to tell when the next forwarding occurs. This means that routing on VHN is difficult in general case.

Therefore we define here a special case of VHN, star-type VHN, on which each node can route packets fairly simply. A star-type VHN is a VHN the nodes of which consist of one router node and other leaf nodes. The router node is connected to all other leaf nodes, and there is no edge between the leaf nodes (Figure 3).

\subsection{PROTOCOLS FOR VHN}

We defined VHN as a protocol independent network structure, but the VHN itself does not work without actual protocol. In this section, we describe two example VHN protocols. One is a reliable point-topoint messaging protocol, and the other is a simple unreliable broadcast protocol. The implementation of a simplified version of the former is described in section 5 . 


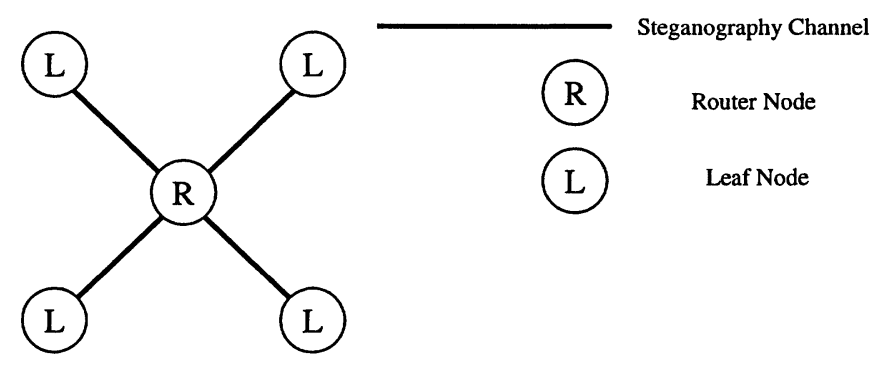

Figure 3 star-type VHN

\section{Example 1: Point-to-Point Protocol over Star-Type VHN}

First we assume that each node has unique ID number and each node knows all the nodes it is connected to. Then there is no routing issue, since the router node can transfer all packets directly to their destination.

In order to provide messaging of big messages regardless of the bandwidth of underlying steganography channel, this protocol supports fragmentation. The format of packet in this protocol is as follows.

$$
\left\langle I D_{\text {from }}, I D_{\text {to }}, S e q, A c k, \text { Len, Data, Csum }\right\rangle
$$

$I D_{\text {from }}$ is the node ID of the sender and $I D_{\text {to }}$ is that of the recipient. $S e q$ is the serial number of this packet and Ack is the serial number of the packet the sender of this packet is waiting for. Len is the length of this packet, Data is a fragment of the message to send, and Checksum is a hash value of the packet except the Checksum part.

When a sender node want to send a message, it first resets the serial number to 0 and precede the message with the length of the message. Next, it fragment the message into packets so that each packet can pass through the steganography channel with a single transaction. Then the sender wait for a cover-data ready to send, and when it is ready, the sender hide the packet into the cover-data with the underlying steganography system. Finally the sender send the resulting stego-data to the routing node. The routing node extracts the packet and stores it. When the steganography channel from the routing node to the recipient node is ready, the routing node forwards the packet to recipient. The recipient node returns acknowledge packet (a packet with 0 length Data and positive Ack 
number set). When the sender sent all messages, sender resends the packet the serial number of which is equal to the highest Ack number received (or 0 if received no $A c k$.) until it receives the acknowledge packet of the last packet it sent. This is actually an fairly simplified version of TCP [8], but it works since no multiplexing is allowed and the router node sees all the packets.

The protocol can be simplified further in order to maximize its efficiency. For example, if the underlying steganography channel is reliable, there is no need for Seq, Ack and Csum field. We can omit $I D_{\text {from }}$ field from the packets directing to router node. We can also omit $I D_{\text {to }}$ field from the packets directing to leaf nodes.

\section{Example 2: Broadcasting Protocol over Generic VHN}

One can construct simple broadcast protocol over VHN by letting each node forward all packets to all its neighbors. In order to prevent packet loop, the following two conditions should be held.

- One should not send the same packet to the same neighbor twice.

- One should not send back a packet to its sender.

The format of packet in this protocol is as follows.

$$
\left\langle I D_{\text {from }}, \text { Serial, Data }\right\rangle
$$

Here $I D_{\text {from }}$ is the originator ID and Serial is a serial number of the packet the originator sends. These fields are necessary to check the identity of the packet.

Since each broadcast packet traverses all edges of the VHN, there arises some redundancy if the VHN is not a tree. The maximum latency of broadcast is proportional to the diameter of the VHN if the steganography channels in the VHN have identical characteristics.

\subsection{SECURITY OF VHN}

The primary purpose of VHN is identical with that of steganography: undetectability. Therefore, security of a VHN can be defined as the difficulty for the eavesdroppers to detect its existence. If it cannot be detected by any eavesdropper, the VHN is perfectly secure. In other words, a public network on which a perfectly secure VHN resides is indistinguishable from the public network without the VHN.

Since the secrecy of VHN traffic solely depends upon ordinary steganography, the security of a VHN is equal to that of the underlying steganography channels. If the underlying steganography is weak, the VHN is 
easy to detect. If the underlying steganography is perfectly secure, the VHN is also perfectly secure.

Unlike the Onion Routing system, VHN is usually vulnerable to internal adversaries. In other words, the existence of VHN packets are not hidden from the nodes in the VHN. It is theoretically possible to hide a message on a VHN by constructing another VHN over the VHN, but it will simply too slow and impractical.

\subsection{ADVANTAGES AND DISADVANTAGES OF VHN}

VHN itself is a useful network structure for people living under oppressive circumstance, since no one can even detect the existence of secure VHN. When compared to ordinary steganography systems, VHN has a significant advantage that it can deliver messages between persons who rarely communicate together or even do not communicate together at all. This feature can be regarded as bandwidth amplification of steganography.

The main disadvantage of VHN is its latency. Since VHN cannot forward the packets on arbitrary time, it can take long time for a packet to be delivered through a VHN. Another disadvantage of VHN is that both sender and recipient should trust all nodes between them. This is not required in original steganography system.

\section{TCP INITIAL SEQUENCE NUMBER CHANNEL}

\subsection{TCP BASICS}

TCP is one of the fundamental protocols on the Internet [8]. TCP provides reliable bidirectional bitstream and most of the common protocols, such as HTTP, SMTP, Telnet, FTP and POP uses TCP as its underlying layer.

The reliability of TCP is achieved by assigning a 32-bit sequence number to each octet it transmits and requiring positive acknowledgment from the recipient of the octets to its sender.

The initial value of the sequence number is called TCP initial sequence number (ISN). When a TCP connection starts between two hosts, each host decides its ISN and announce it to the other host at initial TCP handshake phase. The original TCP specification defines that "the ISN should be sampled from a (possibly fictitious) 32-bit clock whose low order bit is incremented roughly every 4 microseconds." 


\subsection{IP SPOOFING ATTACK AND ISN PREDICTION}

Since TCP itself has no authentication mechanism, it is not difficult to impersonate a host and make a TCP connection to a server if the ISN of the server is predictable to the attacker [2]. Most of old TCP implementations, including those sticking to original TCP specification, have predictable ISN generators and therefore vulnerable to the attack.

Steven Bellovin proposes the following ISN generator as an alternative to original one [2].

ISN $=M+H$ (localhost, localport, remotehost, remoteport, secret-data)

Here $M$ denotes the original 4 microsecond timer and $H$ denotes a cryptographic hash function. The 4-tuple, 〈localhost, localport, remotehost, remoteport $\rangle$, is the set of parameters of the TCP connection establishing.

Most of the ISN generators of recent network operating systems use either Bellovin's generator or its variant, and therefore fairly hard to predict.

\subsection{TCP ISN STEGANOGRAPHY CHANNEL}

In general, any data that depends upon private randomness can be used as a cover data, and it can convey embedded information with perfect secrecy [5]. The randomness of recent ISN generators usually depends on private randomness such as interrupt timings from disks and networks, so ISNs from such generators can be regarded as good cover data. We call this steganography channel TCP initial sequence number channel or TCP ISN channel in short.

Although the bandwidth of this channel is narrow compared to other steganography channels, this channel has several desirable properties. First, most of the network users use TCP connections everyday, so there is no need for changing traffic patterns to use this channel. Secondly, the security of the channel can be guaranteed under the following assumptions.

- The embedding process does not take noticeable amount of time.

- The pseudo random number generator the ISN generator uses are cryptographically secure.

- No adversary can predict the seed for the pseudo random number generator. 
- The embedding data is encrypted with strong cipher and the key for decrypting it is kept secret.

Thirdly, since the embedding and sending process are automatic, the user need not worry about the naturalness of the traffic. Lastly, although the secret information one TCP ISN can carry is small, there are many situations such that many TCP connections are established and discarded again and again. In such cases, this channel can provide practical bandwidth for carrying short messages.

\section{VHN OVER TCP ISN CHANNEL}

We implemented a prototype version of star-type VHN on NetBSD machines. The TCP ISN generator of recent NetBSD is as follows.

$$
\mathrm{ISN}=R+X
$$

Here $X$ denotes various parameters and $R$ denotes an independent 24-bit output from a cryptographically strong pseudo random number generator based on SHA-1 [10]. We use the lower 24-bit of each TCP ISN for secret channel. Since the internal state of the random number generator depends on the interrupt timings from various devices, it is extremely difficult to distinguish the lower 24-bit of each TCP ISN from encrypted messages.

The protocol of our implementation is a variant of the example protocol 1 in section 3.2. Since TCP ISN channel is bidirectional and the recipient of a secret message can send back the same amount of secret information simultaneously, the recipient can always return Ack-flag in synchronous way and therefore $S e q$ and Ack fields are omitted. So the actual packet format is as follows.

$$
\left\langle I D_{\text {from }}, I D_{\text {to }}, \text { Ack-flag, Len, Data, Csum }\right\rangle
$$

Since the TCP ISN channel of NetBSD can deliver only 24 bit at a time, each packet is further divided into 20-bit fragments and padded four 0s on its head. We call this 24-bit fragment "strip." Each strip is encrypted with a stream cipher (Twofish [14] in OFB mode), and hidden into the low order 24 bit of every ISN. The recipient of a packet gathers the strips and rearrange the strips if overtaking occurs (whether overtaking occurred or not can be checked by comparing high order 4 bits of each decrypted strip with "0000".) The Csum is 16-bit one's complement of the one's complement sum of all 16 bit words in the packet. If the recipient can not rearrange the strips so that each high order 4 bits of the decrypted strip is 0 and the Csum field of the decrypted packet is correct, the recipient discards the strips and notify the sender of the 
disposition with a packet with negative acknowledgment flag. Then the sender re-send the original packet again until the recipient send a strip with positive acknowledgment.

The actual implementation consists of three parts: TCP ISN manipulator (a device driver attached to kernel), a daemon program that sends/receives packets in strips, and a userland program for sending or receiving short messages over the VHN. No key manager is implemented yet and therefore the shared secret key for twofish should be distributed prior to the usage of this system. This prototype implementation can send approximately 16 bits per TCP connection.

The safety of this implementation depends on the safety of TCP ISN steganography, the indistinguishability between the low order 24-bit of TCP ISNs of NetBSD and the ciphertext of twofish. Currently both of these have no known vulnerability and it is practically very difficult to distinguish these. Therefore this implementation is practically secure against the eavesdroppers with current technology.

Although the information each TCP ISN can carry is small, many services based on TCP make a lot of TCP connections, and therefore generate a lot of TCP ISNs. For example, most of WWW browsers make many TCP connections to WWW server for downloading images if the downloading page contains many images. If the router node runs WWW server with a lot of images and two persons use WWW browsers to see the images everyday, this implementation can exchange approximately $2 \times$ number of images on the server bytes of secret message between them everyday. If the number of images is large, this VHN can be used as a practical invisible messaging network over the Internet.

\section{CONCLUSIONS}

In this paper, we introduced the notion of Virtual Hidden Network (VHN), an invisible network over public network. It is a network over steganography channels and can amplify the bandwidth of steganographic communication. We next introduced the TCP initial sequence number channel (TCP ISN channel), a steganographic channel that resides in TCP initial sequence numbers. Finally, we introduced our prototype implementation of VHN over TCP ISN channel and showed that VHN over TCP ISN channel can actually construct a practical invisible messaging network over the Internet. 


\section{References}

[1] Ross Anderson, "Stretching the Limits of Steganography," LNCS 1174, Springer, 1996, pp.265-278

[2] S. Bellovin, "Defending Against Sequence Number Attacks," RFC1948

[3] B. Gleeson, A. Lin, J. Heinanen, G. Armitage and A. Malis, "A Framework for IP Based Virtual Private Networks," RFC2764

[4] Information Sciences Institute, University of Southern California, "Internet Protocol," RFC791

[5] Shin NATORI, "One-Time Hash Steganography," LNCS 1768, Springer, 1999, pp.17-28

[6] (Collected by) Birgit Pfitzmann, "Information Hiding Terminology," LNCS 1174, Springer, 1996, pp.347-350

[7] Neil F. Johnson and Sushil Jajodia, "Steganalysis of Images Created Using Current Steganography Software," LNCS 1525, Springer, 1998, pp.32-47

[8] J. Postel, "Transmission Control Protocol," RFC793

[9] Michael G. Reed, Paul F. Syverson, and David M. Goldschlag, "Anonymous Connections and Onion Routing," IEEE Journal on Selected Areas in Communication Special Issue on Copyright and Privacy Protection, 1998

[10] Bruce Schneier, "Applied Cryptography," John Wiley \& Sons, 1996 (second edition)

[11] Bruce Schneier, "Steganography: Truths and Fictions," Crypto-Gram, October 15, 1998, http://www.counterpane.com /crypto-gram-9810.html\#steganography

[12] Gustavus J. Simmons, "The Subliminal Channel and Digital Signatures," EUROCRYPT'84, LNCS 209, Springer, 1985

[13] Andreas Westfeld and Andreas Pfitzmann, "Attacks on Steganographic Systems," LNCS 1768, Springer, 2000, pp.61-76

[14] http://www. counterpane.com/twof ish.html 